\title{
Effect of Various Salts on Monolayer Properties of Partially Substituted Poly(vinyl benzal)s
}

\author{
Masao Koyama and Kenjiro Meguro \\ Department of Chemistry, Faculty of Science, Science University \\ of Tokyo, Kagurazaka, Shinjuku-ku, Tokyo, Japan.
}

(Received December 26, 1973)

\begin{abstract}
The partially substituted poly(vinyl benzal) was synthesized from poly(vinyl alcohol) and benzaldehyde with hydrochloric acid as a catalyst. The change in the surface pressure-area isotherms due to various inorganic and organic salts and urea in the substrate was investigated for the monolayers of the partially substituted poly(vinyl benzal)s by using a modified Wilhelmy balance at $20^{\circ} \mathrm{C}$. The surface pressure-area isotherms of the polymers were expanded progressively with increasing salt concentration. The expansion is in the order: $\mathrm{Na}$ butyrate $>\mathrm{Na}$ benzoate $\gg$ urea $>$ $\mathrm{CaCl}_{2}>\mathrm{BaCl}_{2}>\mathrm{Na}_{2} \mathrm{SO}_{4} \gg \mathrm{NaCl} \simeq \mathrm{KCl}$. It is concluded that the expansion of the monolayer on inorganic salts is caused by the effect of "salting out" and that on organic salts or urea is due to the interactions between the polymers and the solutes in the substrate.
\end{abstract}

KEY WORDS Poly(vinyl benzal) / Poly(vinyl alcohol) / Benzaldehyde / Monolayer / Surface Pressure-Area Isotherms / Salt Effect /

Many authors have chosen to spread proteins and other water-soluble polymers on strong salt solutions such as $10-35 . \%$ ammonium sulfate in water. ${ }^{1-6}$

We found that the monolayers of partially saponified $\operatorname{poly}(n \text {-butyl acrylate })^{7}$ and partially saponified poly(methyl acrylate) $)^{8}$ expanded with increasing salt concentration in the substrate and spread completely on a salt solution of about $15-\% \mathrm{NaCl}$. The monolayers of nonelectrolytic synthetic polymers such as ethoxylin resins ${ }^{9}$ also expanded continuously with increasing ammonium sulfate content in the substrate and spread completely on a substrate of $25-\%$ content. Recently we have demonstrated that the monolayers of poly(vinyl alcohol) which was partially substituted with butyral groups expanded gradually with increasing sodium chloride concentration and spread to definite area on $15 \% .^{10}$

In this investigation, we spread partially substituted poly(vinyl benzal)s on the solutions having different concentration of various salts. The effect of salts and of content of hydrophilic or hydrophobic residues on monolayer properties were discussed.

\section{EXPERIMENTAL}

\section{Preparation of Partially Substituted Poly(vinyl benzal)s}

Poly(vinyl alcohol) purified by reprecipitation from its aqueous solution into methanol was confirmed to be nearly completely saponified (over 99\%) by IR analysis, NMR analysis, and elemental analysis of carbon, hydrogen, and oxygen. The average molecular weight of the poly(vinyl alcohol) was found to be about 79600 by the measurement of viscosity.

Poly(vinyl benzal)s having different degrees of benzal content were prepared by condensation of the poly(vinyl alcohol) with different amounts of benzaldehyde by using hydrochloric acid as a catalyst. The poly(vinyl benzal)s produced were purified by reprecipitation from these dimethylsulfoxide solution into a large excess of distilled water and then dried in vacuo at room temperature. In this work, five polymers were obtained by the condensation method. The benzal content in each polymer was determined by elemental analysis $(\mathrm{C}, \mathrm{H}$, and $\mathrm{O})$ and listed in Table I.

The poly(vinyl benzal)s contain both hydrophilic (vinyl alcohol) and hydrophobic (vinyl 
Table I. Degree of benzal content ${ }^{a}$ and limiting area of partially substituted poly(vinyl benzal)s

\begin{tabular}{lcc}
\hline & $\begin{array}{c}\text { Degree of } \\
\text { benzal content, } \\
\text { mol\% }\end{array}$ & $\begin{array}{c}\text { Limiting area on } \\
\text { distilled water, } \\
\AA^{2} / \text { residue }\end{array}$ \\
\hline PVBe20 & 21.49 & 13.4 \\
PVBe30 & 33.46 & 17.4 \\
PVBe50 & 52.00 & 23.3 \\
PVBe70 & 67.09 & 28.6 \\
PVBe80 & 77.49 & 30.2 \\
\hline
\end{tabular}

a Polymer analisis by elemental determination of $\mathrm{C}, \mathrm{H}$, and $\mathrm{O}$.

benzal) portions such as in following schematic formula.

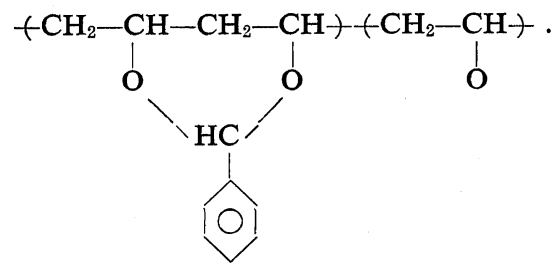

Since there are two groupings of hydrophilic and hydrophobic portions in the polymer, it is expected that the polymer molecules orient themselves at the air-water interface so as to give a monolayer with hydroxyl groups immersed in the substrate and phenyl groups in the air.

\section{Surface Pressure Measurement}

A modified Wilhelmy-type surface balance with glass plate was used to measure the surface pressure. The surface balance was completely enclosed in a thermostatted box. The copperalloy trough and glass barriers were carefully coated with paraffin wax and washed with running water to remove the trace of the surfaceactive impurities on the paraffin wax. The apparatus was kept at constant temperature $(20 \pm$ $0.5^{\circ} \mathrm{C}$ ) by circulating the water from thermostatted bath through the trough jacket.

The water used as substrate was deionized and distilled from alkaline potassium permanganate in an all glass still. The salts dissolved in substrate water were reagent grade $\mathrm{KCl}$, $\mathrm{NaCl}, \mathrm{Na}_{2} \mathrm{SO}_{4}, \mathrm{CaCl}_{2} \cdot 2 \mathrm{H}_{2} \mathrm{O}, \mathrm{BaCl}_{2} \cdot 2 \mathrm{H}_{2} \mathrm{O}, \mathrm{Na}$ butyrate, $\mathrm{Na}$ benzoate and urea. When the surface of the substrate was swept with barriers, the surface pressure values during blank runs (i.e., in the absence of a monolayer) coincided within experimental error. These blank runs showed that there were no detectable surfaceactive impurities in the salt solutions.

The monolayers were spread on various substrate solutions from a mixed solvent consisting of benzene/dimethylsulfoxide (60/40, volume/ volume). After spreading the polymer, thirty minutes were allowed for the solvent to evaporate and then the compression of the film was started. The measurement of the surface pressure was done at $5 \mathrm{~min}$ after the stop of every short compression to be reached equilibrium. The barrier was driven at a rate of $1.0 \AA^{2}$ per residue per minute. The surface pressure-area measurement was repeated several times, the results were reproducible within experimental error.

\section{RESULTS AND DISCUSSION}

The surface pressure-area isotherms of poly(vinyl benzal) (PVBe80) containing 77.49-mol\% benzal residues at different concentrations of sodium chloride in the substrate are shown in Figure 1. As the sodium chloride concentration becomes higher, the monolayer tends to be more expanded. Similar behavior was observed for the monolayers of partially substituted poly(vinyl butyral)s $\mathrm{s}^{10}$ and of others. ${ }^{7-9}$

When the compressibility was plotted against area, the curve had a minimum at the area corresponding to the point of inflection in the lower surface pressure of the surface pressure of the surface pressure-area isotherm. After passing through this point, the isotherm had a linear portion. The limiting area could be obtained by extrapolation of the linear portion to a surface pressure of zero. The limiting area increased from $30.2 \AA^{2}$ per residue in curve 1 to $33.2 \AA^{2}$ per residue in curve 3 with increasing sodium chloride concentration. All of the poly(vinyl benzal)s showed the same expansion.

Since the poly(vinyl benzal) which is substituted partially with benzal groups has both vinyl alcohol and benzal residues, some hydrophilic portions having hydroxyl groups may be immersed in the water by the interaction with 


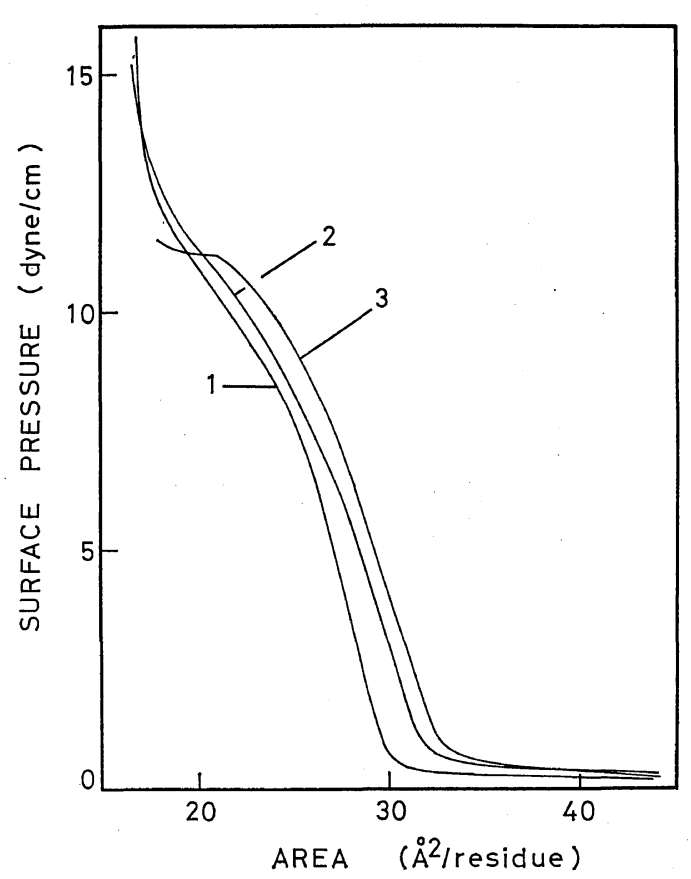

Figure 1. Surface pressure-area isotherms of polymer PVBe80 on distilled water (1), 0.5-M $\mathrm{NaCl}(2)$, and $1.0-M \mathrm{NaCl}(3)$ at $20^{\circ} \mathrm{C}$.

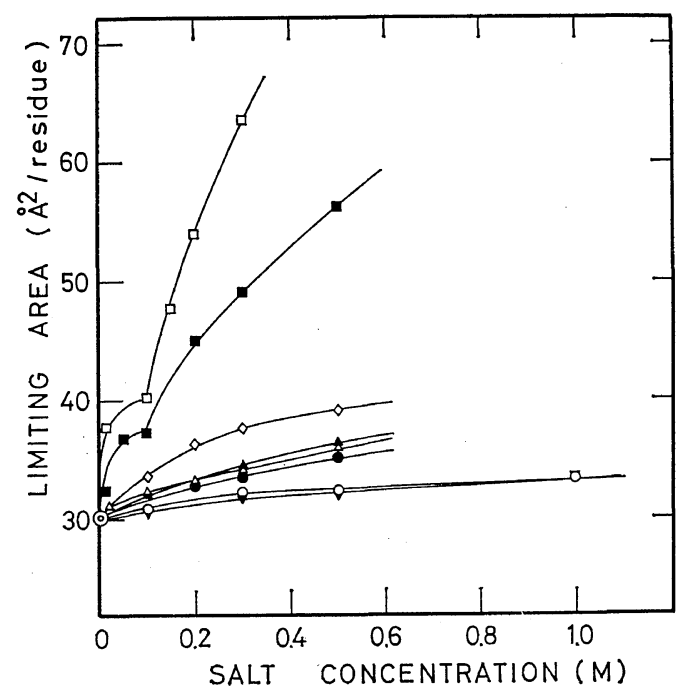

Figure 2. Limiting areas of polymer PVBe80 as a function of the concentration for various salts at $20^{\circ} \mathrm{C}: \square$, Na butyrate; $\square, \mathrm{Na}$ benzoate; $\diamond$, urea; $\triangle, \mathrm{CaCl}_{2} ; \triangle, \mathrm{BaCl}_{2} ; \bigcirc, \mathrm{Na}_{2} \mathrm{SO}_{4} ; \bigcirc, \mathrm{NaCl}$; $\nabla, \mathrm{KCl}$. water molecules. Therefore, increasing the sodium chloride concentration in the substrate forced out the hydrophilic portions on to the substrate surface by a salting-out effect. That is to say, it is considered that the expansion of the monolayer due to an increase in the salt concentration is caused by the effect of saltingout. This concept is supported by many reports. $^{7-11}$ The monolayers of the polymers containing both hydrophilic and hydrophobic portions, such as partially saponified polymers ${ }^{7,8}$ and ethoxylin resin $^{9}$ and partially substituted poly(vinyl butyral)s, ${ }^{10}$ spread completely on strong salt solutions. This is explained by the assumption that the hydrophilic portions submerging into the substrate are squeezed out on the surface by salting-out effect. A similar salting-out effect has been reported for the film of polyoxyethylene $n$-dodecanol on sodium chloride solution by Lange. ${ }^{11}$ The increase in area at high salt concentrations would also result from a decrease in immersion of hydrophilic polyoxyethylene chains accompanying a reduction of hydration of these chains.

A remarkable expansion was also observed in the case of the monolayers on the solution of $\mathrm{KCl}, \mathrm{Na}_{2} \mathrm{SO}_{4}, \mathrm{BaCl}_{2}$, and $\mathrm{CaCl}_{2}$. The monolayers of $\mathrm{PVBe} 80$ on these salt solutions expanded progressively with increasing salt concentration as summerized in Figure 2. As shown in Figure 2, the degree of the expansion of PVBe80 monolayer may be dependent upon ionic size or charge. For the $0.5-M$ salt solutions, the PVBe 80 monolayer is expanded as shown in Figure 3, and the expansion follows the order:

$$
\mathrm{CaCl}_{2}>\mathrm{BaCl}_{2}>\mathrm{Na}_{2} \mathrm{SO}_{4} \gg \mathrm{NaCl} \approx \mathrm{KCl}
$$

This order is similar to that of the Hofmeister series for metal chlorides and identical with that found for the expansion of the simple long chain alcohol, such as $n$-octadecanol, as reported by Ralston, et $a{ }^{12}{ }^{12}$ It is very interesting that the expansion of a nonelectrolytic polymer film such as these partially substituted poly(vinyl benzal)s follows the lyotropic series.

When the substrate contains organic salts such as sodium butyrate and sodium benzoate, the monolayer of PVBe80 expands tremendously. Since the organic salts have surface activity, it 


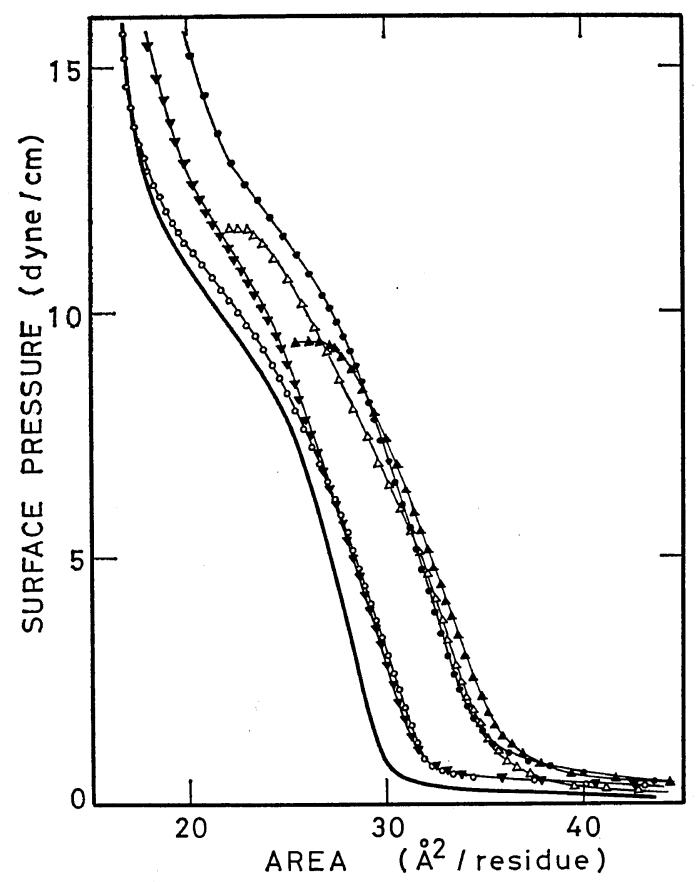

Figure 3. Surface pressure-area isotherms of polymer PVBe80 on distilled water and a series of $0.5-M$ inorganic salt solutions at $20^{\circ} \mathrm{C}$ : $\nabla$, $\mathrm{KCl} ; \bigcirc, \mathrm{NaCl} ; \bigcirc, \mathrm{Na}_{2} \mathrm{SO}_{4} ; \triangle, \mathrm{BaCl}_{2} ; \Delta, \mathrm{CaCl}_{2}$.

is necessary to estimate the surface pressure of the organic salt solutions. The result showed that the surface pressure was little changed in any of the compression region. However, the addition of the organic salt in the substrate had an effect on the expansion of the polymer monolayer as shown in Figures 4 and 5. It is considered that the organic salts are fixed in the monolayer by the interaction with the polymer.

The poly(vinyl benzal) used in this investigation has both hydrophilic and hydrophobic groups in the molecule. The hydrophobic phenyl groups in the polymer may interact strongly with benzoate molecules in the substrate and the benzoate molecules should penetrate into the poly(vinyl benzal) monolayer. Therefore, the limiting area increases to about twice on $0.5-M$ sodium benzoate solution. The monolayer on sodium butyrate solution was also expanded tremendously. It is considered that the butyrate molecules are also fixed in the polymer monolayer by the interaction with the polymer

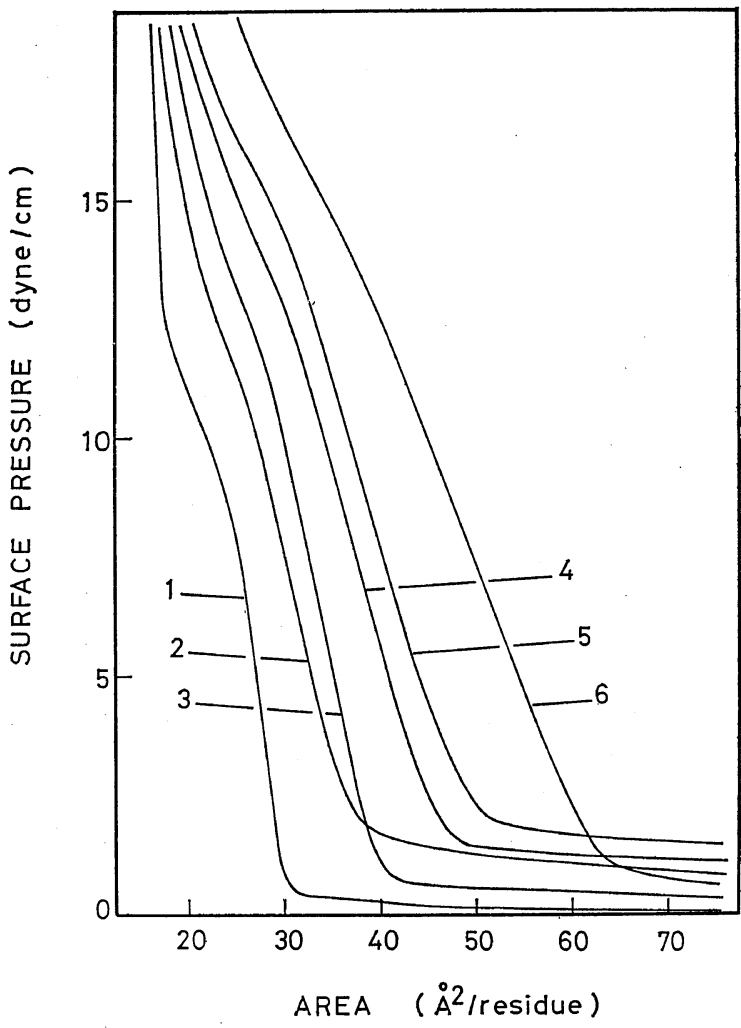

Figure 4. Surface pressure-area isotherms of polymer $\mathrm{PVBe} 80$ on substrates containing various amounts of sodium butyrate at $20^{\circ} \mathrm{C}$ : 1 , distilled water; $2,0.01 M ; 3,0.1 M ; 4,0.15 M ; 0.2 M ; 6$, $0.3 \mathrm{M}$.

molecules. The difference in expansion of the films on two organic salt solutions is considered to be caused by the difference in cohesive forces between the polymer films and the organic salts. As the cohesive force between the phenyl groups seemed not only to be strong but also to be greater than that between the polymer and butyrate molecules, the monolayer on sodium butyrate solution may be more expanded than the film on sodium benzoate solution.

A remarkable expansion was also found in the monolayer on urea solution as shown in Figure 6. This would also be due to the reduction of hydration of hydrophilic groups by adding urea. On the other hand, the addition of urea in the substrate appears to raise the surface pressure of inflection, which lies above the linear portion in the surface pressure-area isotherm. 


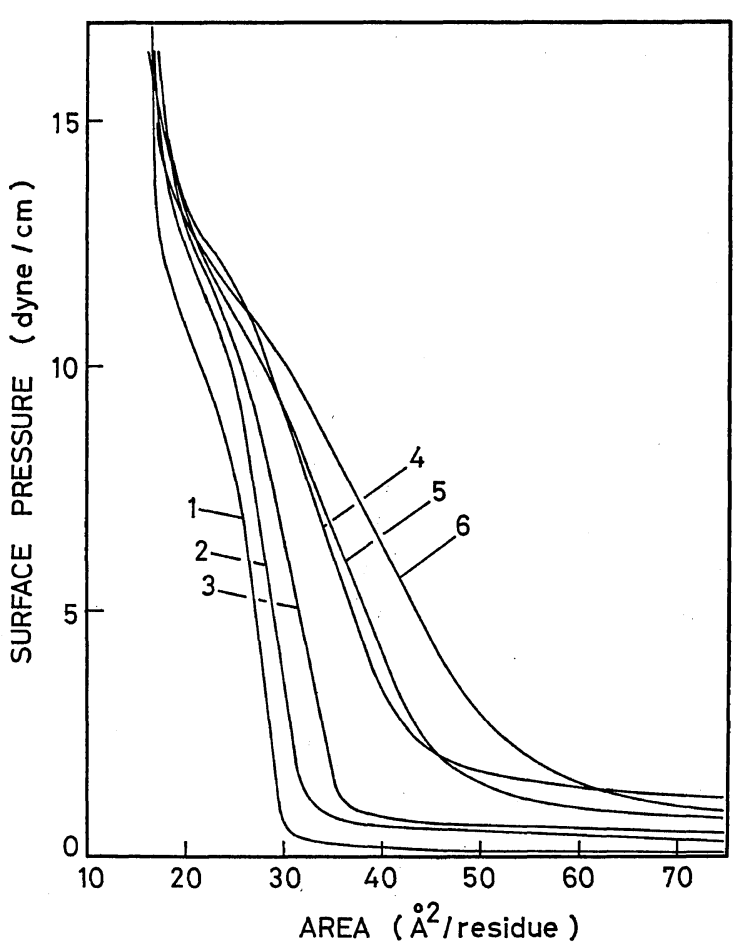

Figure 5. Surface pressure-area isotherms of polymer PVBe80 on substrates containing various amounts of sodium benzoate at $20^{\circ} \mathrm{C}: 1$, distilled water; $2,0.01 M ; 3,0.05 M ; 4,0.2 M ; 5,0.3 M$; $6,0.5 \mathrm{M}$.

The appearance of the inflection may be due to the rearrangement of segmental orientation in the film. When the polymer film on water is compressed above the inflection, it becomes gellike probably as a result of the formation of hydrogen bonding between the water molecules and the polymer. If the salts or urea in the substrate compete with the polymer for the water molecules, the interaction between the polymer and the water molecules, such as hydrogen bonding, would be weakened by the increase in the salt concentration of the substrate. Consequently, the gelation of the polymer film would be prevented up to higher pressures. As shown in Figures 3 and 6, the increase in surface pressure of the inflection by urea appears to be greater than that by other inorganic salts. It suggests that the rearrangement of segmental orientation is disturbed more by urea than by inorganic salts since urea upsets the hydrogen

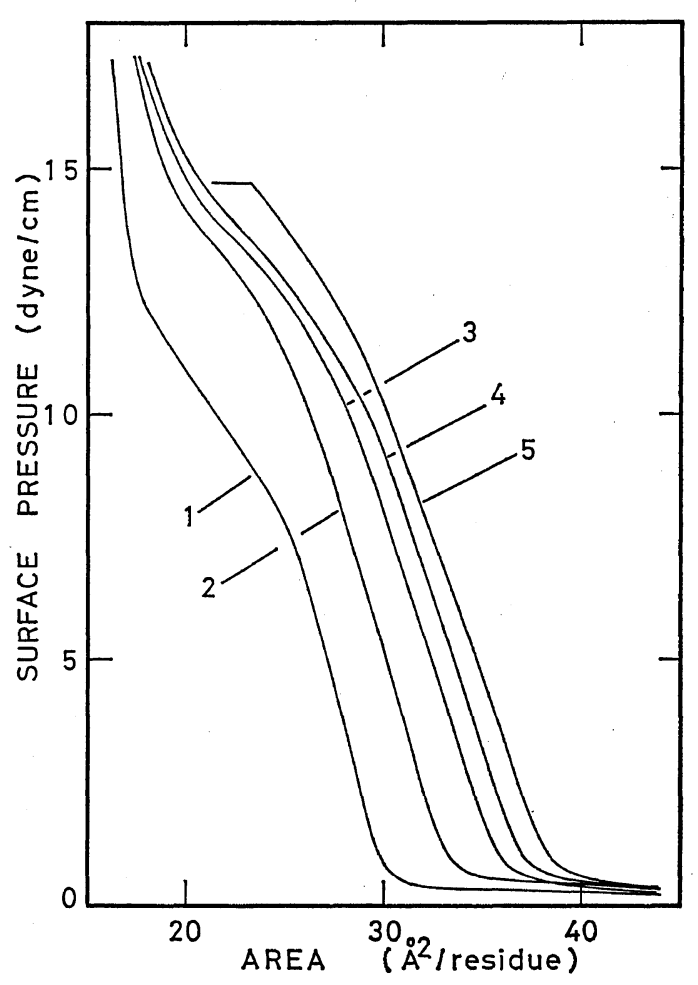

Figure 6. Surface pressure-area isotherms of polymer PVBe80 on substrates containing various amounts of urea at $20^{\circ} \mathrm{C}: 1$, distilled water; 2 , $0.1 M ; 3,0.2 M ; 4,0.3 M ; 5,0.5 M$.

bonding between the water molecules and the polymer. Sears ${ }^{13}$ showed that the increase in surface pressure of stearic and palmitic acid monolayers was caused by the penetration of urea into an interface from the substrate depending upon the dipole-dipole interaction between urea and fatty acid molecules. In our investigation, urea may weaken the hydrogen bonding between water molecules in the substrate and hydroxyl groups in the partially substituted poly(vinyl benzal).

Figure 7 shows the relationship between the benzal content in the polymer and the increment in the limiting area by addition of the salt. Here, $\Delta A$ is equal to the difference between the limiting area on $0.5-M$ salt solution and that on distilled water. As shown in Figure 7 , the expansion by sodium chloride is hardly changed by the change in polymer composition, that is, the expansion caused by the addition 


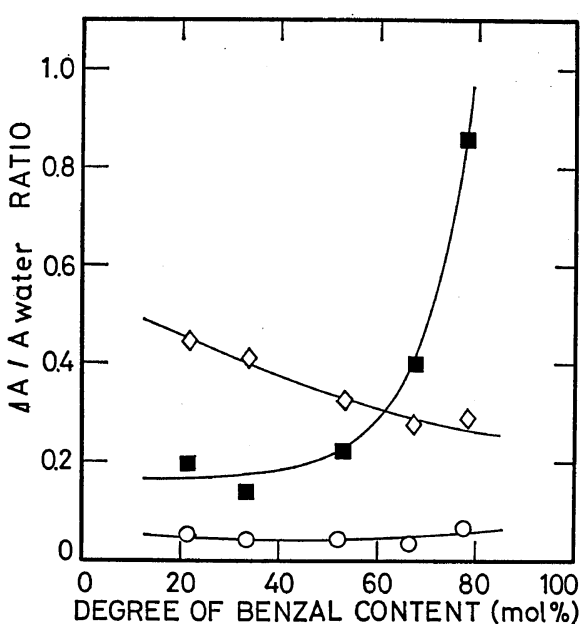

Figure 7. Ratio between the limiting area increment $(\triangle A)$ and limiting area for distilled water ( $A_{\text {water }}$ ) at $0.5 \mathrm{M}$ and $20^{\circ} \mathrm{C}$ as a function of the degree of benzal content: $\square$, $\mathrm{Na}$ benzoate; $\diamond$, urea; $\mathrm{O}, \mathrm{NaCl}$.

of inorganic salts is nearly constant regardless of the change in the degree of benzal content. The expansion by inorganic salts may be caused by only an ionic strength.

On the other hand, the monolayer on sodium benzoate solution showed a steep expansion with increasing benzal content. Since the sodium benzoate molecules are expected to interact with phenyl groups in the poly(vinyl benzal), the benzoate molecules would presumably penetrate into the monolayers of the partially substituted poly(vinyl benzal) at the air-water interface.

In turn, for the film on urea solution the ratio of areas decreases gradually with the increase in benzal content, that is, the polymer containing may hydroxyl groups is more ex- panded than that having lower hydroxyl content at a given constant concentration of urea. Therefore, it is found that the interaction between water molecules and hydroxyl groups in the polymer is decreased by the urea in the substrate.

Acknowledgment. The authors wish to thank Mr. M. Ueno for kindly advice and Miss $M$. Yamamoto and Mr. Y. Tomioka for assistance with the measurements.

\section{REFERENCES}

1. A. J. G. Allan and A. E. Alexander, Trans. Faraday Soc., 46, 316 (1950).

2. B. D. Powell and A. E. Alexander, J. Colloid Sci., 7, 482 (1952).

3. E. Mishuck and F. R. Eirich, J. Polym. Sci., 16, 397 (1955).

4. H. B. Bull, J. Amer. Chem. Soc., 67, 4, 8 (1945).

5. J. Miñones Trillo, E. Iribarnegaray Jado, S. García Fernández, and P. Sanz Pedrero, Kolloid-Z. Z. Polym., 250, 325 (1972).

6. J. Miñones, S. García Fernández, E. Iribarnegaray, and P. Sanz Pedrero, J. Colloid Interfac. Sci., 42, 503 (1973).

7. S. Hironaka and K. Meguro, ibid., 35, 367 (1971).

8. S. Hironaka and K. Meguro, Bull. Chem. Soc. Japan, 45, 332 (1972).

9. J. Glazer, J. Polym. Sci., 13, 355 (1954).

10. M. Koyama, R. Tomioka, M. Ueno, and K. Meguro, Kolloid-Z. Z. Polym., in press.

11. H. Lange in "Nonionic Surfactants", M. J. Schick, Ed., Marcel Dekker, Inc., New York, N.Y., 1967, p 443.

12. J. Ralston and T. W. Healy, J. Colloid Interfac. Sci., 42, 629 (1973).

13. D. F. Sears, ibid., 29, 288 (1969). 